\title{
Titanium in silicate glasses and melts
}

ThiBAut Robine ${ }^{1}$, CHARLES Le LosQ ${ }^{1}$, DANiEl R. NEUVILlE ${ }^{1}$

${ }^{1}$ Laboratoire de géomatériaux - Institut de physique du globe de Paris (IPGP) - CNRS : UMR7154 - 75005 Paris -

France

Titanium has a key role in both materials and Earth sciences. For instance, the presence of $\mathrm{TiO} 2$ enhances the glass refractive index and improve mechanical properties. Moreover, small amounts of $\mathrm{TiO} 2$ in the glass bulk can be added in order to induce homogeneous crystallization.

In geosciences, the study of $\mathrm{Ti}$ valence is critical. Typically, $\mathrm{Ti}$ is mainly stable as tetravalent and acts as network former within the glass network; however, in planetary materials, it can be largely stabilised as trivalent. Hence, the $\mathrm{Ti} 4+/ \mathrm{Ti} 3+$ redox ratio is believed to be a key parameter to assess the formation conditions of certain planetary materials, especially in $\mathrm{Ca}$ and $\mathrm{Al}$ rich inclusions, usually considered to be the oldest solid materials of our solar system. However, the quantification of the redox condition of these bodies could be challenging, since the parameters mostly affecting the $\mathrm{Ti} 4+/ \mathrm{Ti} 3+$ redox ratio, and $\mathrm{Ti}$ coordination environment (including bonding type) are not fully constrained.

In this study, we investigated a series of amorphous, molten and crystalline compounds in order to ascertain to what extent bulk composition, oxygen fugacity and temperature influence titanium speciation. 